\title{
Reduction in the Viability of Human Cervical Cancer HeLa Cell Line via Indirect Co-culture With Amniotic Fluid-Derived Mesenchymal Stem Cells
}

\author{
Faramarz Rahmatizadeh ${ }^{1,2,3}{ }^{\circledR}$, Fatima Pashaei-Asl ${ }^{4}$, Manijeh Mohammadi Dehcheshmeh ${ }^{5}$, Sara Rahbar ${ }^{6}$, \\ Maryam LaleAtaei ${ }^{2,7}$, Shiva Gholizadeh-Ghaleh Aziz ${ }^{8^{*} \mathbb{D}}$, Jafar Soleimani $\operatorname{Rad}^{7,9 *}$, Maryam Pashaias $^{3,7,9,10^{*}}$
}

\begin{abstract}
Objectives: This experiment was carried out to evaluate the impacts of unmodified human amniotic fluid-derived mesenchymal stromal/stem cells (hAF-MSCs) on the viability of HeLa cells, as well as the impact of these cells on the expression of common proapoptotic and pro-survival genes in tumor cells by establishing an indirect co-culture system.

Materials and Methods: To this end, an indirect co-culture system was established, and hAF-MSCs were co-cultured with HeLa cells at a ratio of 1:2 for five days. The cell viability of co-cultured tumor cells was determined after the incubation period. Then, several parameters were examined, including the gene expression of tumor protein 53 (TP53), BCL2-associated X protein (BAX), B-cell lymphoma 2 (BCL-2), and cyclin-dependent kinase inhibitor 1A (CDKN1A). Finally, gene regulatory networks were analyzed as well.

Results: The results of this study confirmed that the co-culture of hAF-MSCs with HeLa cells could decrease the viability of tumor cells. The reduction of HeLa cell viability was accompanied by an increase in BAX, TP53, and CDKN1A while a decrease in BCL2 gene expression. Eventually, the analysis of the regulatory network revealed that the co-culture of Hela $\neg$ cells with hAF-MSCs activated several transcriptional factors and microRNAs which regulated the expression of these genes.

Conclusions: In general, hAF-MSCs exerted the inhibitive effects on the growth of HeLa cells, along with alterations in the expression of common pro-apoptotic and pro-survival genes in a timely and concentration-dependent manner.

Keywords: Mesenchymal stem cells, HeLa cells, Amniotic fluid, Co-culture, Cell-based therapy
\end{abstract}

\section{Introduction}

Cervical cancer is a group of cancer that originates from the entrance to the uterus, so-called as the uterine cervix. There are several lines of evidence indicating that the rates of incidence and mortality of cervical cancer cases can be controlled and prevented through comprehensive and integrated approaches. In addition, it is the fourth most frequently diagnosed cancer, and the second major cause of malignancy-related mortality among women globally $(1,2)$. Moreover, deaths which occur due to cervical cancer in developing countries continue to be one of the most important causes of all malignancy-related mortalities. The widespread lack of accessibility to cervical cancer screening tests including Papanicolaou's (Pap) smear and human papillomavirus (HPV) testing for women susceptible to this malignancy is the main cause of cervical cancer-related mortality in these countries (3).

The incidence rate with an estimation of 570000 women afflicted with this malignancy was recorded worldwide in 2018 representing $7.5 \%$ of all female cancers (4). Although the incidence rate of cervical cancer was strikingly low in Iran in 2012, its mortality rate was high. The obtained data from global cancer statistics-GLOBOCAN 2012 presented the estimates of the incidence rate of cervical cancer as 2.8 in 2012 and a death rate of 1.2 per 100000 per year (5). The incidence and mortality rates varied across different geographic regions of Iran and relied on different factors. The findings showed that the early age of first pregnancy, smoking, early age at marriage, use of oral contraceptive pills, family history, and multiple sexual partners are

\footnotetext{
Received 10 May 2019, Accepted 9 September 2019, Available online 28 September 2019

${ }^{1}$ Department of Molecular Medicine, Faculty of Advanced Medical Science, Tabriz University of Medical Science, Tabriz, Iran. ${ }^{2}$ Student Research Committee, Tabriz University of Medical Sciences, Tabriz, Iran. ${ }^{3}$ Drug Applied Research Center, Tabriz University of Medical Sciences, Tabriz, Iran. ${ }^{4}$ Molecular Biology Laboratory, Biotechnology Research Center, Tabriz University of Medical Sciences, Tabriz, Iran. ${ }^{5}$ School of Animal and Veterinary Sciences, the University of Adelaide, Adelaide, Australia. ${ }^{6}$ Department of Reproductive Biology and Anatomical Sciences, Faculty of Medicine, Shahid Beheshti University of Medical Sciences, Tehran, Iran. ${ }^{7}$ Department of Anatomical Sciences, Faculty of Medicine, Tabriz University of Medical Sciences, Tabriz, Iran. ${ }^{8}$ Department of Clinical Biochemistry, Faculty of Medicine, Urmia University of Medical Sciences, Urmia, Iran. ${ }^{9}$ Department of Reproductive Biology, Faculty of Advanced Medical Science, Tabriz University of Medical Science, Tabriz, Iran. ${ }^{10}$ Women's Reproductive Health Research Center, Tabriz University of Medical Sciences Tabriz, Iran.

*Corresponding Authors: Shiva Gholizadeh-Ghaleh Aziz, Email: gholizadeh.sh@umsu.ac.ir; Jafar Soleimani Rad, Email: soleimanirj@yahoo.com; Maryam Pashaiasl, Email: Pashaim@tbzmed.ac.ir
} 
Key messages

- In this study, we showed the suppressive effects of human amniotic fluid-derived mesenchymal stem cells (hAFMSCs) on the human cervical cancer HeLa cell line.

- The growth of cervical cancer HeLa cells can be inhibited in the concentration and time-dependant manner by hAFMSCs.

among the major risk factors that are associated with the incidence and death rates of cervical cancer in Iran (6).

Many types of HPV have been detected in many different groups of species, some of which have been exclusively found in human beings (7). However, HPV infections can lead to cervical cancer in some cases (8). It was estimated that 79.1 million people were infected with HPVs (also known as the most prevalent sexually transmitted infection) in 2008. It is noteworthy that oncogenic HPV types have been detected in more than $99 \%$ of cases of invasive cervical cancer worldwide (9). This malignancy usually occurs following persistent infections with oncogenic HPV types, especially high-risk HPV (hrHPV) types 16, 18, 31, and 45 (10).

The latest research findings have indicated that the hrHPVs encode two oncoproteins of E6 and E7 which are responsible for the malignant transformation of human cervical epithelial cells (11). In other words, the main stages of the pathogenic mechanism of this malignancy include the binding of HPV E6 and E7 to p53 and retinoblastoma tumor suppressor protein $(\mathrm{pRB})$ subsequently stimulate the proteasomal degradation of p53 and pRB, respectively (12).

Patients suffering from cervical cancer usually have no noticeable manifestations in the early stages of the disease. However, they can undergo medical check-ups at regular intervals for the early diagnosis of cervical cancer.

The choice of standard treatment options for cervical cancer often depends on clinical stages that include traditional surgical excision in the early stages of the disease. Meanwhile, the most common treatment options include radiation therapy and chemotherapy in the advanced stages of cervical cancer. It is noteworthy that $80 \%-95 \%$ of women with early-stage cervical cancer can be cured with chemoradiotherapy and surgery. However, the metastatic and recurrent disease remains the main cause of cancerrelated deaths, and patients with metastatic cervical cancer have unfavorable prognosis with the median survival of 8-13 months. In addition, cancer chemotherapeutic drugs are rarely curative and are considered as palliative care options $(13,14)$. The development of new approaches and paradigms in diagnostic, therapeutic, and prognostic strategies should also be taken into consideration which is essential for attaining an upper level of clinical cancer management (15).

Recently, different areas of research have paid particular attention to the role of two early oncoproteins (i.e., HPV E6 and E7) which are important in transforming the lining cells of the cervix. These oncoproteins are consistently expressed and retained in tumor cells which are necessary for the initial induction and maintenance of the malignant phenotype.

In recent decades, research studies have heavily focused on other molecular targeted drugs, therapeutic vaccines (16), hyperthermia (17), sentinel lymph node biopsy (18), and immunotherapy approaches (19). Although these therapeutic approaches for cervical cancer treatment are not yet approved for clinical use, many studies have focused on these procedures.

Mesenchymal stem cells hold remarkable promise for the application of cancer cell-based therapies. Several recent studies have shown that mesenchymal stromal/ stem cells (MSCs) exhibit intrinsic anti-tumor properties against Kaposi's sarcoma (20), glioblastoma (21), Burkitt's lymphoma (22), pancreatic cancer (23), and breast cancer (24). However, the suppressive effects of MSCs on breast cancer still have been incompletely conceptualized (25). The underlying mechanisms responsible for the anti-tumor effects of unmodified MSCs include the induction of apoptosis in tumor cells, inhibition of cell cycle progression, and the inhibition of specific signaling pathways (e.g., PI3K/Akt and Wnt/ $\beta$-catenin signaling transduction pathways). In addition, other mechanisms such the inhibition of tumor angiogenesis and neovascularization, induction of mesenchymal-toepithelial transition, suppressive effects on cancer stem cells, a significant impact on immune responses such as the ability to increase inflammatory infiltrations, and the deactivation of the X-chromosome-linked inhibitor of apoptosis protein expression have been observed within tumor cells $(20,26,27)$.

Many findings suggest that important underlying mechanisms responsible for MSC-mediated antiproliferative effects on Hela cells consist of the inhibition of mitogen-activated protein kinases (MAPKs) and PI3K signaling pathways in tumor cells. The findings have also revealed that the involvement of MAPK-signaling pathways is a turning point for the cellular reaction to p53 activation, apoptosis, and cell cycle arrest (28).

Nowadays, there has been growing attention to human amniotic fluid-derived mesenchymal stromal/stem cells (hAF-MSCs) that can be amenable to cell-based therapies $(29,30)$. The possibility of isolating a large number of these cells, and avoiding moral hazard problems, as well as having multipotent differentiation property, self-renewal ability, and extensive proliferation capacity are the main advantages for clinical use of these cells. In general, these particular characteristics render hAF-MSCs suitable for cell-based therapies $(2,31)$.

Previous research indicated that the direct or indirect co-culture of tumor cells with MSCs or culturing tumor cells with MSC-derived conditioned media can affect 
the development of tumor cells (28). Further, evidence suggested that unmodified (intact) MSCs from different sources have the opposite divergent effects on tumor growth and progression. Therefore, the therapeutic effects of hAF-MSCs should be evaluated when faced with different types of cancer.

Accordingly, the main objective of the current investigation was to assess the impacts of hAF-MSCs on the viability of HeLa cells by using an indirect co-culture system with respect to determined cell concentration and incubation period. To the best of our knowledge, no previous study has evaluated this issue. Nonetheless, the effects of this co-culture on common pro-apoptotic and pro-survival genes have been assessed using the quantitative polymerase chain reaction. The regulatory network analysis of miRNAs and transcriptional factors was performed using Pathway Studio software.

Unmodified hAF-MSCs are a promising solution for cell-based cancer therapies and may develop novel anticancer strategies against human cervical cancer.

\section{Materials and Methods}

Primary Cells, Cell Culture Media, and Supplements Human Amniotic Fluid-Derived Mesenchymal Stromal/ Stem Cells

In our previous studies, hAF-MSCs were successfully isolated by using the single-step method of isolation (33). The isolated cells were identified and then moved into a liquid nitrogen storage tank until further use. The cryopreserved hAF-MSCs were then thawed, resuspended, and plated in a $25 \mathrm{~cm}^{2}$ cell culture flask at a concentration of $1 \times 10^{4}$ cells $/ \mathrm{cm}^{2}$. Thereafter, $3-5 \mathrm{~mL}$ of pre-warmed $\left(37^{\circ} \mathrm{C}\right)$ low glucose Dulbecco's modified Eagle's medium (LDMEM) supplemented with 10\% fetal bovine serum (FBS, Sigma Aldrich), a $10 \mathrm{ng} / \mathrm{mL}$ basic fibroblast growth factor (bFGF, Sigma Aldrich), 1\% L-glutamine, and a 1\% (v/v) penicillin/streptomycin (Pen/Strep) solution were added to the culture medium. The cultured cells were maintained in an incubator in a humidified atmosphere with $5 \% \mathrm{CO}_{2}$ at $37^{\circ} \mathrm{C}$. Moreover, the culture medium was replaced twice per week and the cells were left to grow for four to five days until reaching a confluency of $70 \%$. Finally, hAF-MSCs were sub-cultured, and 0.05\% (w/v) Trypsin/0.02\% (w/v) EDTA (Sigma Aldrich, USA) was used for the detachment of cells. All experiments were performed using hAF-MSCs from passages 4 to 6 .

\section{HeLa Cell Line}

The human cervical adenocarcinoma cell line HeLa (HeLa cell line, NCBI_Iran, cat\# CD115, RRID: CVCL_0030) was prepared from the National Cell Bank of Iran (NCBI), Pasteur Institute of Iran (IPI-Tehran). The HeLa cells were plated in a cell culture flask with a surface area of $25 \mathrm{~cm}^{2}$, and the DMEM culture medium (Sigma Aldrich) supplemented with 5\% FBS (Sigma Aldrich) and 1\% Pen/ Strep (Sigma Aldrich) solution were then added to the culture. The cells were then placed in a $5 \% \mathrm{CO}_{2}$ incubator at $37^{\circ} \mathrm{C}$.

Human Fibroblast Cell Line

Human fetal foreskin fibroblasts (HFFF2 cell line, NCBI Iran, cat\# C163, RRID: CBCL_2489) were obtained from the NCBI, IPI-Tehran and then cultivated in DMEM cell culture media supplemented with $10 \%$ FBS (Sigma Aldrich) at $37^{\circ} \mathrm{C}$ in a $5 \% \mathrm{CO}_{2}$ incubator.

\section{Experimental Design and Setup}

In this study, experimental groups composed of HeLa cells. The viability of HeLa cells and common pro-apoptotic and pro-survival gene expression levels were evaluated on the 3 rd and 5th day of the co-culture with hAF-MSCs. HeLa cells in the indirect co-culture system at passage four were plated onto a 24 -well cell culture plate in triplicate, and $0.6 \mathrm{~mL}$ of culture media per well was added in the lower compartment as we explained earlier (32).

For the indirect co-culture between hAF-MSCs and HeLa cells, Transwell ${ }^{\circledR}$ tissue culture plate inserts with a pore size of $0.4 \mu \mathrm{m}$ (6.5 mm membrane diameter, Corning) were used to evaluate the impacts of hAF-MSCs on HeLa cells. In this regard, an indirect co-culture system was applied to provide a condition in which a microporous membrane with a pore size of $0.4 \mu \mathrm{m}$ could be used to separate two cell populations so that each cell population had its own culture-specific condition. Therefore, indirect intercellular communication could take place mostly via soluble molecules.

The hAF-MSCs were seeded onto the upper compartment, then $0.01 \mathrm{~mL}$ of DMEM complete media was added to the culture. The ratio number of hAF-MSCs vs. HeLa cells was 1:2 according to literature methods. Previous research findings demonstrated that the antitumor effects of MSCs rely on their incubation periods and concentrations. Thus, the selection of sample-size was based on the information that was described in the literature (34-36).

In addition, this experimental study also assessed the impacts of stem cells on HeLa cells, meanwhile the ratio number of hAF-MSCs vs. HeLa cells was 1:2 and the duration of the incubation period was three days. All groups were tested in triplicate under the same experimental conditions. The control groups were composed of untreated HeLa cells which were not cocultured with hAF-MSCs whereas the comparator control groups included fibroblasts that were co-cultured with HeLa cells under the same experimental conditions.

MTT (3-(4,5-Dimethylthiazol-2-yl)-2,5-diphenyltetrazolium bromide) Cell Viability Assay

As previously explained, the viability of HeLa cells was measured by the MTT-based assay on the third and fifth days of co-culture with hAF-MSCs (36). Briefly, HeLa cells were cultured and then maintained in an incubator at 
$37^{\circ} \mathrm{C}$ under $5 \% \mathrm{CO}_{2}$ in a humidified atmosphere. After the incubation period, the upper media were removed and the MTT solution ( $5 \mathrm{mg} / \mathrm{mL}$, Sigma-Aldrich) was added to the wells. Next, the cells were maintained for four hours in an incubator at $37^{\circ} \mathrm{C}$ as well. Thereafter, the MTT solution was discarded and Dimethyl sulfoxide (DMSO, Tokyo, Japan) was poured into each respective well in order to dissolve the purple formazan crystals. The absorbance of each respective well was read at a wavelength of $570 \mathrm{~nm}$ using a multi-well plate reader.

Quantitative Real-Time (qRT) Reverse Transcription Polymerase Chain Reaction (PCR)

RT-PCR was performed as described before $(33,36)$. Briefly, the total cellular RNA was extracted using the RNXPlus solution (CinnaGen, Iran, Tehran cat\# RN7713C) according to the manufacturer's recommendations, and assessed via the Nanodrop ND-2000 spectrophotometer in order to confirm the purity of RNA (Thermo Fisher Scientific). The cDNA Synthesis Kit (Fermentas, cat\# K1621) was also used to synthesize cDNA from the extracted RNA. It was prepared at the final volume of $20 \mu \mathrm{L}$ for each sample in a Thermal Cycler (PEQLAB, Erlangen, Germany). Thereafter, the qRT-PCR amplification was carried out using the SYBR Green 1 dye method for validating candidate gene expression $(45,46)$. In this regard, SYBR ${ }^{\circledR}$ Premix Ex TaqTM II, cat\#RR820 (Takara, Bio) was used based on the manufacturer's instructions. The qPCR was conducted with the RT-PCR system (MIC, Switzerland with the following parameters: incubation at $94^{\circ} \mathrm{C}$ for 5 minutes, followed by 40 cycles of denaturation at $95^{\circ} \mathrm{C}$ for 30 seconds, annealing at $60^{\circ} \mathrm{C}$ for 30 seconds, and extension at $72^{\circ} \mathrm{C}$ for 30 seconds). The experiments were performed based on primer pair sequences as presented in Table 1. The results were normalized to $\beta$-actin mRNA expression patterns which were employed as accurate internal control genes. The relative gene expression of candidate genes including TP53, BAX, BCL2 , and CDKN1A was calculated by the $2^{-\Delta \Delta C t}$ method. All experiments were carried out in triplicate.

Regulatory Network Analysis of Up-Regulated ProApoptotic BAX, CDKN1A, and TP53, and Pro-Survival BCL-2 Genes After Co-Culture

Regulatory network analysis was designed based on literature mining using the Pathway Studio tool (Elsevier) as discussed previously (37-40). To this end, the upregulated pro-apoptotic BAX, CDKN1A, TP53, and prosurvival BCL-2 genes after the co-culture were used as the input, and relationships were retrieved from the literature based on the relationship interaction of regulation, mRNA-effect, and promoter binding.

\section{Statistical Analysis}

All numerical (quantitative) data were expressed as the mean \pm standard deviation (SD). Statistically significant
Table 1. The Primer Pair Sequences Used for Quantitative Real-time Polymerase Chain Reaction and Their Appropriate Annealing Temperatures

\begin{tabular}{|c|c|c|}
\hline Gene & Primer & $\begin{array}{c}\text { Annealing } \\
\text { Temperatures }\left({ }^{\circ} \mathrm{C}\right)\end{array}$ \\
\hline$\beta$-Actin & $\begin{array}{l}\text { FWD: 5? } \text { ? }-A A A T C T G G C A C C A C A C C T T C \\
\text { REV: 5? } \text { ?-CCATCTCTTGCTCGAAGTCC }\end{array}$ & $49^{\circ} \mathrm{C}$ for $45 \mathrm{~s}$ \\
\hline OCT4 & $\begin{array}{l}\text { FWD: 5?-AGGTGTTCAGCCAAACGACC } \\
\text { REV: 5?-TGATCGTTTGCCCTTCTGGC }\end{array}$ & $60^{\circ} \mathrm{C}$ for $30 \mathrm{~s}$ \\
\hline NANOG & $\begin{array}{l}\text { FWD: 5?]- ATCCAGCTTGTCCCCAAAG } \\
\text { REV: 5?]- ATTTCATTCTGGTTCTGG }\end{array}$ & $60^{\circ} \mathrm{C}$ for $30 \mathrm{~s}$ \\
\hline BAX & $\begin{array}{l}\text { FWD:5?-TGCTACAAGGTTTCATTTAG } \\
\text { REV: 5? } \text { ? ATCCACATCAGCAATCATCC }\end{array}$ & $59^{\circ} \mathrm{C}$ for $30 \mathrm{~s}$ \\
\hline BCL-2 & $\begin{array}{l}\text { FWD: 5?- ACTTTGCAGAGATGTCCAGT } \\
\text { REV: 5?- CGGTTCAGGTACTCAGCAT }\end{array}$ & $55^{\circ} \mathrm{C}$ for $30 \mathrm{~s}$ \\
\hline TP53 & $\begin{array}{l}\text { FWD: 5?-TGCTGAGTATCTGGACGACA } \\
\text { REV: 5? - GAGCGTGATGATGGTAAGG }\end{array}$ & $59^{\circ} \mathrm{C}$ for $30 \mathrm{~s}$ \\
\hline CDKN1A & $\begin{array}{l}\text { FWD: 5?]- GCGATGGAACTTCGACTTTGT } \\
\text { REV: 5?]- GGTAGAAATCTGTCATGCTGGTC }\end{array}$ & $58^{\circ} \mathrm{C}$ for $30 \mathrm{~s}$ \\
\hline C-MYC & $\begin{array}{l}\text { FWD:5?-GCTCAAAGCCTAACCTCACAA } \\
\text { REV:5?AAAGAAAGAAGATGGGAAGCA }\end{array}$ & $59^{\circ} \mathrm{C}$ for $30 \mathrm{~s}$ \\
\hline
\end{tabular}

differences among experimental and non-experimental groups were calculated by the one-way analysis of variance (one-way ANOVA) and the Tukey's HSD test (posthoc-test when necessary). Eventually, the statistical data analysis was carried out using GraphPad Prism software, version 7.0 (GraphPad Inc., San Diego, California, USA), and a $P<0.05$ was considered statistically significant.

\section{Results}

The Reduction in the Viability of HeLa Cells

The measurement of HeLa cell viability using the MTT assay indicated that HeLa cell viability was obviously attenuated after indirect co-culture with hAF-MSCs at $37^{\circ} \mathrm{C}$ in a $5 \% \mathrm{CO}_{2}$ incubator for five days (1:2 ratio of hAFMSCs to HeLa cells) when the results of experimental co-culture groups were compared to those of the control groups. The obtained results were statistically significant $(P<0.05)$ (Figure 1).

However, no statistically significant changes were found in the viability of HeLa cells after three days of incubation with hAF-MSCs at a ratio of 1:2 hAF-MSCs/HeLa cells in an indirect co-culture (the results are not presented).

\section{Pluripotency Gene Expression}

The mRNA levels of pluripotency genes (i.e., NANOG, OCT4, and C-MYC) in hAF-MSCs after five days of an indirect co-culture between hAF-MSCs and HeLa cells remained elevated at around the same level before coculture between HeLa cells and hAF-MSCs $(P>0.05)$ (Figure 2).

The mRNA Expression Levels of Pro-Apoptotic and ProSurvival Genes

The indirect co-culture of hAF-MSCs with HeLa cells at a ratio of 1:2 and an incubation period of five days (in a $5 \% \mathrm{CO}_{2}$ incubator at a temperature of $37^{\circ} \mathrm{C}$ ) led to the up- 


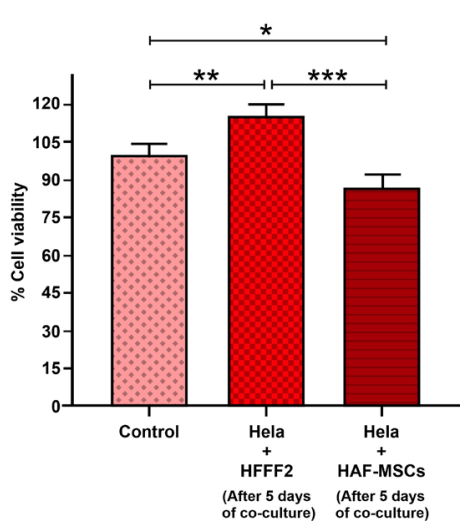

Figure 1. HeLa cell viability in experimental groups were measured by MTT cell viability assay and then compared with the data from the control groups: ${ }^{*} P-=0.0163,{ }^{* *} P=0.0095,{ }^{* *} P=0.0004$; hAF-MSCs: Human amniotic fluid-derived mesenchymal stromal/stem cells; HFFF2: Human fetal foreskin fibroblasts.

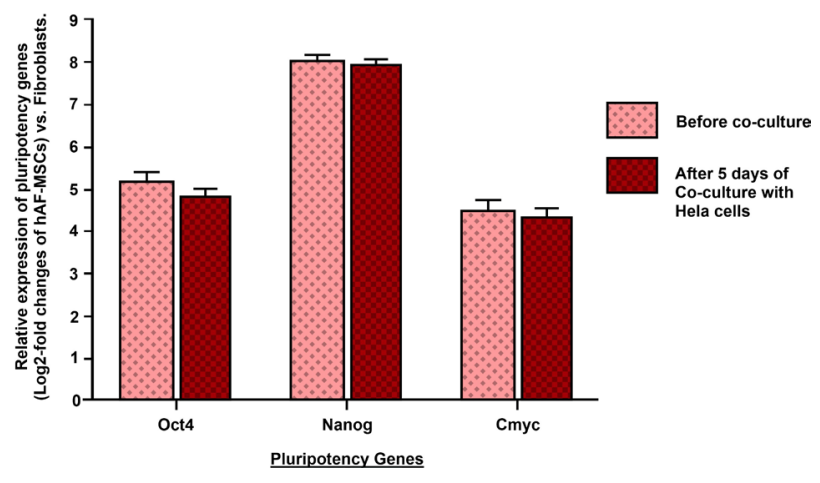

Figure 2. Expression levels of the pluripotency markers of hAF-MSCs before and after indirect co-culture with HeLa cells were compared with the control group (composed of HFFF2). hAF-MSCs: Human amniotic fluidderived mesenchymal stromal/stem cells; HFFF2: Human fetal foreskin fibroblasts.

regulation of TP53 $(P<0.0001)$, CDKN1A $(P<0.0001)$, and $\operatorname{BAX}(P=0.0001)$ in comparison to that of the control groups. In the meantime, the analysis of the basal expression levels of frequent pro-apoptotic genes (i.e., TP53, CDKN1A, and BAX) showed a significant increase in tumor cells while the expression level of the pro-survival BCL-2 gene decreased compared to the control groups $(P=0.0006)$. Figure 3 illustrates the mRNA expression levels of candidate pro-survival BCL-2 and pro-apoptotic CDKN1A, BAX, and TP53 genes in HeLa cells after coculture with hAF-MSCs.

There were no statistically significant differences among the results of experimental co-culture groups compared to those of the control groups $(P>0.05)$ when hAF-MSCs were indirectly co-cultured with HeLa cells at a ratio of 1:2 after three days of incubation.

\section{An Elevated BAX/BCL-2 Ratio}

The indirect co-culture of hAF-MSCs with HeLa cells at 1:2 hAF-MSCs vs. HeLa cells ratio with an incubation period of five days resulted in a significantly higher BAX/
BCL 2 expression ratio $(P<0.0001)$ than that of the control groups (Figure 4).

The Analysis of Regulatory Network

Network analysis showed that co-culture activates a combination of transcription factors and miRNAs that regulate BAX, CDKN1A, TP53, and BCL-2. The regulatory transcription factors were SOX2, Sp1, KLF4, POU4F1, EGR1, TP53, TP73, RELA, NR3C1, TBX2, MYC, BCL6, and E2F1. At the miRNA level, many apoptosis-related candidate miRNAs were MiR181B1, MiR20A, MiR19B1, MiR21, MiR22, MiR221, MiR214, MiR181A1, and metastasis-associated candidates were MALAT1, MiR372, MiR23A, MiRLET7A1, MiR222, and MiR296. Two protein kinases ofMAP2K7 and STK4 were involved in network regulation. The extracted relations are depicted in Figure 5.

\section{Discussion}

Our findings indicated that the viability of HeLa cells can be decreased in an indirect co-culture system by using an appropriate proportion of hAF-MSCs to HeLa cells, along with optimal incubation time. After 5 days of incubation at a 1:2 ratio of hAF-MSCs/HeLa cells, there was a remarkable decrease in the HeLa cell viability which was accompanied by the up-regulation of the mRNA expression levels of pro-apoptotic CDKN1A, BAX, and TP53, as well as the down-regulation of pro-survival $B C L-2$ genes. Furthermore, these data revealed that the co-culture of hAF-MSCs with HeLa cells could lead to a significant augmentation in the BAX/BCL-2 ratio.

In the current study, the effects of hAF-MSCs on HeLa cells were determined after co-culture between hAF-MSCs and HeLa cells. Nowadays, hAF-MSCs hold great promise as a formidable source of stem cells that is amenable to cell-based therapies $(41,42)$. These cells possess a high replicative potential, the capability to differentiate into all three germ-layers or lineages, and the ability to maintain their stemness and multipotency for long periods of time. In addition, these cells can be readily extracted from the amniotic fluid and can be isolated and purified in large amounts.

Despite the controversy surrounding current diversity in MSC-based regenerative therapy and promising perspectives for the future of this field, there has been an emerging area of research which focuses on the development of new MSC-based therapeutic approaches in order to improve clinical results in patients with cancer (43). It is noteworthy that unmodified MSCs exert bidirectional effects on tumor progression via different mechanisms. It means that these cells exhibit both antiand pro-tumor activities. Moreover, the findings suggest that several conditions and factors alter the pro- or antitumor activities of MSCs. For instance, MSCs exert dual effects on tumor growth in a concentration-dependent manner. Thus, MSCs have anti- and pro-tumor activities 

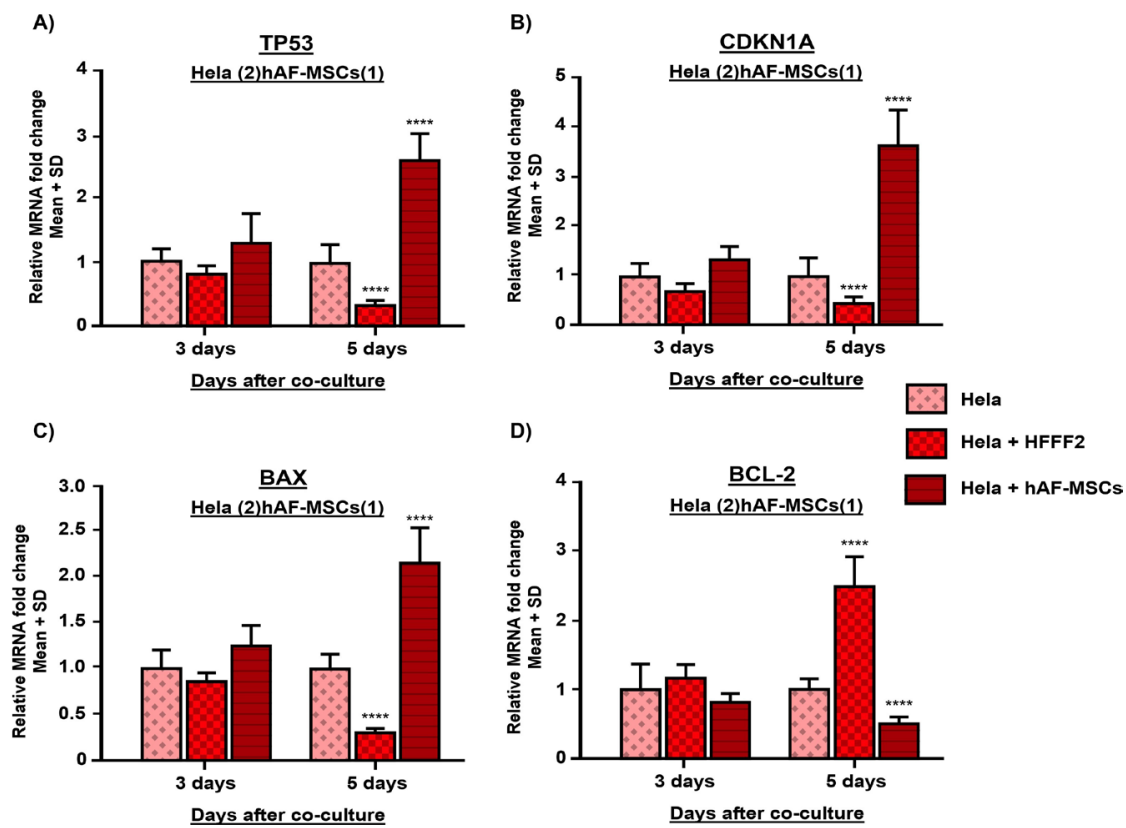

Figure 3. The mRNA expression levels of pro-apoptotic BAX, CDKN1A, and TP53 and pro-survival BCL-2 genes were measured both in experimental and control groups and then were compared against each other. (A) TP53, (B) CDKN1A, (C) BAX, (D) BCL-2. ${ }^{* * *} P=0.0001,{ }^{* * * *} P<0.0001$; hAF-MSCs: Human amniotic fluid-derived mesenchymal stromal/stem cells; HFFF2: Human fetal foreskin fibroblasts.

which rely on the ratio of MSCs to tumor cells. Similarly, time-course experiments have demonstrated that MSCs exert time-dependent effects on tumor growth (44). Our experiments were performed in a 1:2 ratio number of hAF-MSCs vs. HeLa cells with an incubation period for five days of co-culture (35). The cells were examined on the third and fifth days of co-culture.

Several empirical studies have highlighted that MSCs can have pro- or anti-tumor effects through cell-cell communications, indirect interactions, and when tumor cells are cultured with mesenchymal stem cell-derived conditioned media $(45,46)$. This study aimed to establish an indirect co-culture model to reveal that hAF-MSCs play a pivotal role in decreasing the viability of HeLa cells.

In addition, the current study determined a significant alteration in pro-apoptotic BAX, CDKN1A, TP53, and

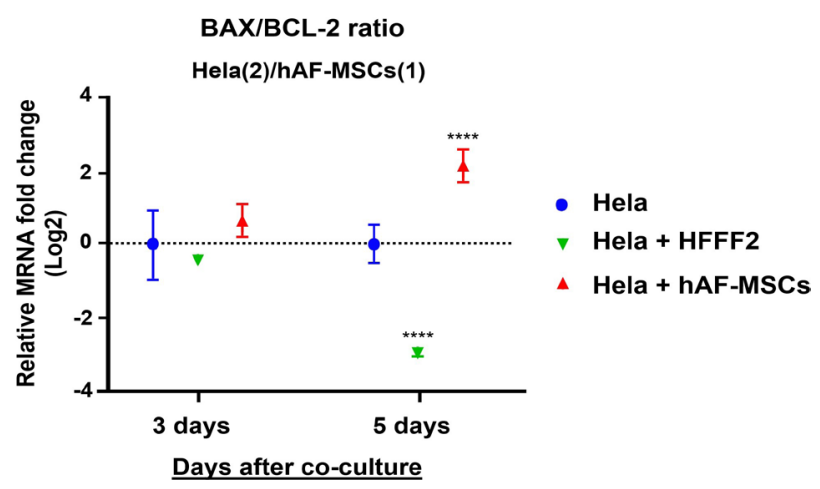

Figure 4. The determination of $B A X / B C L-2$ ratio in HeLa cells on the third and fifth day in indirect co-culture with hAF-MSCs at a 1:2 ratio of hAFMSCs/HeLa cells; hAF-MSCs: Human amniotic fluid-derived mesenchymal stromal/stem cells; HFFF2: Human fetal foreskin fibroblasts. pro-survival BCL-2 gene expression. Indeed, the loss of tumor-suppressing functions of p53 in HeLa cells results in the enhancement of tumor cell proliferation.

There is strong evidence for the existence of a direct causal relationship between persistent HPV infection and cervical cancer pathogenesis in more than $99.7 \%$ of all cases (9). Persistent HPV infection is the main, but not the only risk factor for women afflicted with cervical cancer (3). In addition to the HPV infection, there are many other risk factors which are related to this malignancy that can increase the risk of acquiring HPV or persistent high-risk HPV infection, including a weakened immune system, exposure to mutagens, and hormonal factors. Meanwhile, genetic predisposition (genetic susceptibility) is found to play a subordinate role in the progression of cervical cancer. The other well-known and important risk factors which can enhance their susceptibility to cervical cancer include the presence of more than one sexual partner, the early commencement of the sexual activity, association with sexually transmitted infections, cigarette smoking, oral contraceptive use, pregnancy, and the family history, as well as women whose mothers took diethylstilbestrol during pregnancy and people with low socioeconomic status $(47,48)$.

Vaccination against HPV can effectively prevent the infections due to oncogenic HPV types which are responsible for nearly $70 \%$ of all $\mathrm{HPV}$-associated genital diseases and cervical cancer among eligible candidates (3).

The hrHPV oncoproteins of E6 and E7 have a decisive role in the natural history of cervical HPV infection and the molecular pathogenesis of cervical cancer. After conjugation with ubiquitin-protein ligase E6-AP, the 


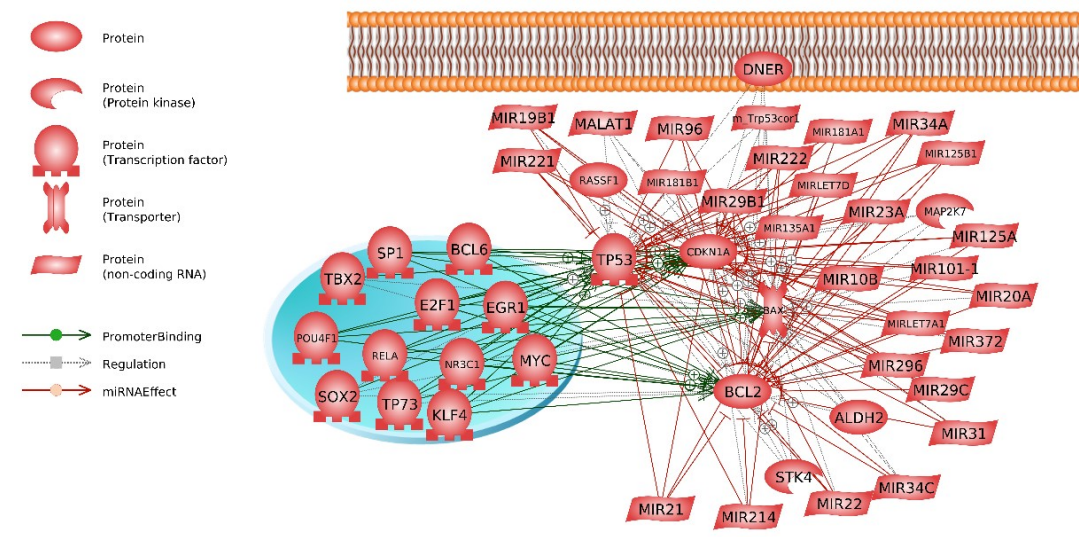

Figure 5. Regulatory network analysis of pro-apoptotic BAX, CDKN1A, TP53, and pro-survival BCL-2 genes after co-culture.

E6 oncoprotein can target p53 for ubiquitination and subsequently its proteasomal degradation whereas the E7 oncoprotein can interact with $\mathrm{pRB}$ protein and facilitate its proteasomal degradation (49). Generally, the persistent hrHPV infection of the lining cells of the uterine cervix can alter molecular signature in the affected cells $(49,50)$.

The increased TP53 expression level in HeLa cells after co-culture with hAF-MSCs led to the restoration of the p53 function. This field of study still requires more in-depth investigations to obtain better results. It was demonstrated that p53 has the ability to transactive downstream target genes by the up-regulation of CDKN1A, MDM2, and cell cycle-regulated gene expression at their transcriptional levels. Several recent studies have shown that many transcriptional targets can be efficiently mediated by p53 activities. Additionally, more than one hundred genes have so far been identified that can be directly targeted by $553(51,52)$. Moreover, the expression of the CDKN1A gene is tightly controlled by p53 transactivation activity. Naturally, P21 $1^{\text {cip1/waf1 }}$ blocks the G1-to-S phase transition of the cell cycle through the inhibition of D-type cyclindependent kinases (i.e., CDK4 and CDK6) and the dephosphorylation of $\mathrm{pRB}$ (which is the biologically active form of $\mathrm{Rb}$ protein). In addition, p53 tumor-suppressor protein interacts with the pro-survival members of the $\mathrm{Bcl}-2$ family proteins (e.g., $\mathrm{Bcl}-\mathrm{xL}$ and $\mathrm{Bcl}-\mathrm{w}$ ).

The findings of the present study suggested that the BAX mRNA expression level in HeLa cells was up-regulated after five days of co-culture with hAF-MSCs whereas the BCL-2 gene expression level was down-regulated at the same time point. It is remarkable that the presence of p21 is required for Bax liberation in order to suppress the tumor cell invasion and induction of apoptosis. Indeed, the generation of $\mathrm{p} 53 / \mathrm{p} 21 / \mathrm{Bcl}-\mathrm{w}$ complex maintains the relationship among all couples such as p21/Bcl-w and p53/p21. In addition, reciprocal interactions between p53 and $\mathrm{Bcl}-\mathrm{w}$ are required for $\mathrm{p} 53$ activity in order to directly bind to BCL-w that finally leads to the liberation of Bax from the complex.

Besides, the $\mathrm{Bax} / \mathrm{Bcl}-2$ expression ratio can be used to determine the susceptibility of cells to apoptosis and can actually act as a molecular rheostat. According to this concept, it is more realistic to assume that a high $\mathrm{Bax} / \mathrm{Bcl}-2$ ratio is characteristic of the sensitivity of cells to apoptosis. Meanwhile, a decrease in $\mathrm{Bax} / \mathrm{Bcl}-2$ ratio is highly positively correlated with the cell resistance to apoptosis. Hence, measuring the expression ratios of pro-apoptotic BAX to anti-apoptotic BCL-2 genes is more crucial for cell fate determination compared to measuring each of them individually (53). Our findings further suggested that the BAX mRNA expression level was markedly up-regulated while simultaneously the BCL-2 gene expression was down-regulated in HeLa cells after five days of indirect co-culture with hAF-MSCs.

Regulatory network analysis demonstrated the involvement of key apoptotic transcription factors, noncoding RNAs, and protein kinases in the regulation of the upregulated genes after co-culture.

The obtained data showed that the clinical use of hAFMSCs might be a promising choice for improving the treatment of cervical cancer. However, a great effort has been devoted to identify a strategy for controlling the underlying mechanisms which are responsible for the divergent and dual effects of hAF-MSCs on the growth and progression of tumors in order to optimize the outcomes of cell-based therapies.

One suggestion for future research directions in this field of study is the identification of conditions and factors which have an impact on hAF-MSCs to exert dual effects on the growth and development of tumors. In addition, the polarization of hAF-MSCs via toll-like receptors which augment their anti-tumor effects and can generate the anti-tumor phenotypes such as MSC1, and the conflicting role of hAF-MSCs in different molecular pathways which could enhance the tumor-suppressive effects of these cells can be considered as the other suggestions in this regard.

\section{Conclusions}

In the present study, we implemented the Guiding Principles underlying previous studies which focused 
on the interactions between MSCs and tumor cells. It was experimentally demonstrated that hAF-MSCs can suppress the HeLa cell growth in an indirect co-culture system which was accompanied by alterations in the mRNA expression levels of common pro-apoptotic and pro-survival genes by applying previous experimental outcomes.

\section{Conflict of Interests}

Authors declare that they have no conflict of interests.

\section{Ethical Issues}

This empirical research was approved by the Institutional Review Board and the Ethics Committee of Tabriz University of Medical Sciences, Tabriz, IRAN (Ethical Code: 5/Dal /8713).

\section{Financial Support}

Drug Applied Research Center,Tabriz University of Medical Sciences supported the study.

\section{Acknowledgments}

The authors would like to express their deepest gratitude and appreciation to the staff of the Department of Molecular Medicine (Tabriz), Faculty of Advanced Medical Sciences, Tabriz University of Medical Sciences (TUOMS), and the Drug Applied Research Center at TUOMS, Tabriz University of Medical Sciences, TabrizIran whose assistance proved to be a milestone in the accomplishment of this project.

\section{References}

1. Siegel RL, Miller KD, Jemal A. Cancer statistics, 2018. CA Cancer J Clin. 2018;68(1):7-30. doi:10.3322/caac. 21442

2. Cervical cancer [Internet]. NIH Fact Sheets. National Institutes of Health (NIH); 2018.

3. Chauhan SC, Jaggi M, Bell MC, Verma M, Kumar D. Epidemiology of human papilloma virus (HPV) in cervical mucosa. Methods Mol Biol. 2009;471:439-456. doi:10.1007/978-1-59745-416-2_22

4. Cervical cancer. https://www.who.int/cancer/cervicalcancer. World Health Organization; 2018.

5. 5Ferlay J, Soerjomataram I, Dikshit R, et al. Cancer incidence and mortality worldwide: sources, methods and major patterns in GLOBOCAN 2012. Int J Cancer. 2015;136(5):E359-386. doi:10.1002/ijc.29210

6. Momenimovahed Z, Salehiniya H. Cervical cancer in Iran: integrative insights of epidemiological analysis. Biomedicine (Taipei). 2018;8(3):18. doi:10.1051/bmdcn/2018080318

7. Cervical cancer: Human papillomaviruses (HPV). Institute for Quality and Efficiency in Health Care (IQWiG). Cologne, Germany: 2006. 2017 Dec 14. Available from: https://www. ncbi.nlm.nih.gov/books/NBK279260/.

8. Baseman JG, Koutsky LA. The epidemiology of human papillomavirus infections. J Clin Virol. 2005;32 Suppl 1:S1624. doi:10.1016/j.jcv.2004.12.008

9. Alexander KA, Giuliano AR. HPV-beyond cervical cancer (online resource center). Am J Med. 2012;125(7):S1. doi:10.1016/j.amjmed.2012.03.005
10. Clifford GM, Smith JS, Aguado T, Franceschi S. Comparison of HPV type distribution in high-grade cervical lesions and cervical cancer: a meta-analysis. Br J Cancer. 2003;89(1):101105. doi:10.1038/sj.bjc.6601024

11. Park EK, Kim YW, Lee JM, et al. Immunization with adenoviral vectors carrying recombinant IL-12 and E7 enhanced the antitumor immunity against human papillomavirus 16-associated tumor. Cancer Res Treat. 2005;37(1):63-70. doi:10.4143/crt.2005.37.1.63

12. Crook T, Tidy JA, Vousden KH. Degradation of p53 can be targeted by HPV E6 sequences distinct from those required for p53 binding and trans-activation. Cell. 1991;67(3):547556. doi:10.1016/0092-8674(91)90529-8

13. Petignat P, Roy M. Diagnosis and management of cervical cancer. BMJ. 2007;335(7623):765-768. doi:10.1136/ bmj.39337.615197.80

14. Li H, Wu X, Cheng X. Advances in diagnosis and treatment of metastatic cervical cancer. J Gynecol Oncol. 2016;27(4):e43. doi:10.3802/jgo.2016.27.e43

15. van Meir H, Kenter GG, Burggraaf J, et al. The need for improvement of the treatment of advanced and metastatic cervical cancer, the rationale for combined chemo-immunotherapy. Anticancer Agents Med Chem. 2014;14(2):190-203.

16. Cordeiro MN, De Lima RCP, Paolini F, et al. Current research into novel therapeutic vaccines against cervical cancer. Expert Rev Anticancer Ther. 2018;18(4):365-376. doi:10.10 80/14737140.2018.1445527

17. Jha S, Sharma PK, Malviya R. Hyperthermia: role and risk factor for cancer treatment. Achievements in the Life Sciences. 2016;10(2):161-167. doi:10.1016/j.als.2016.11.004

18. Bader AA, Winter R, Haas J, Tamussino KF. Where to look for the sentinel lymph node in cervical cancer. Am J Obstet Gynecol. 2007;197(6):678.e671-677. doi:10.1016/j. ajog.2007.09.053

19. Orbegoso C, Murali K, Banerjee S. The current status of immunotherapy for cervical cancer. Rep Pract Oncol Radiother. 2018;23(6):580-588. doi:10.1016/j. rpor.2018.05.001

20. Khakoo AY, Pati S, Anderson SA, et al. Human mesenchymal stem cells exert potent antitumorigenic effects in a model of Kaposi's sarcoma. J Exp Med. 2006;203(5):1235-1247. doi:10.1084/jem.20051921

21. Breznik B, Motaln H, Vittori M, Rotter A, Lah Turnsek T. Mesenchymal stem cells differentially affect the invasion of distinct glioblastoma cell lines. Oncotarget. 2017;8(15):25482-25499. doi:10.18632/oncotarget.16041

22. Lin HD, Fong CY, Biswas A, Choolani M, Bongso A. Human Wharton's jelly stem cells, its conditioned medium and cellfree lysate inhibit the growth of human lymphoma cells. Stem Cell Rev. 2014;10(4):573-586. doi:10.1007/s12015-0149514-3

23. Kidd S, Caldwell L, Dietrich M, et al. Mesenchymal stromal cells alone or expressing interferon-beta suppress pancreatic tumors in vivo, an effect countered by antiinflammatory treatment. Cytotherapy. 2010;12(5):615-625. doi:10.3109/14653241003631815

24. He N, Kong Y, Lei X, et al. MSCs inhibit tumor progression and enhance radiosensitivity of breast cancer cells by down-regulating Stat3 signaling pathway. Cell Death Dis. 2018;9(10):1026. doi:10.1038/s41419-018-0949-3

25. Usha L, Rao G, Christopherson Ii K, Xu X. Mesenchymal stem cells develop tumor tropism but do not accelerate breast cancer tumorigenesis in a somatic mouse breast cancer 
model. PLoS One. 2013;8(9):e67895. doi:10.1371/journal. pone.0067895

26. Rhee KJ, Lee JI, Eom YW. Mesenchymal stem cell-mediated effects of tumor support or suppression. Int J Mol Sci. 2015;16(12):30015-30033. doi:10.3390/ijms161226215

27. Dasari VR, Velpula KK, Kaur K, et al. Cord blood stem cellmediated induction of apoptosis in glioma downregulates $\mathrm{X}$-linked inhibitor of apoptosis protein (XIAP). PLoS One. 2010;5(7):e11813. doi:10.1371/journal.pone.0011813

28. Long X, Matsumoto R, Yang P, Uemura T. Effect of human mesenchymal stem cells on the growth of HepG2 and HeLa cells. Cell Struct Funct. 2013;38(1):109-121.

29. Zhou J, Wang D, Liang T, Guo Q, Zhang G. Amniotic fluid-derived mesenchymal stem cells: characteristics and therapeutic applications. Arch Gynecol Obstet. 2014;290(2):223-231. doi:10.1007/s00404-014-3231-7

30. Gholizadeh-Ghalehaziz S, Farahzadi R, Fathi E, Pashaiasl M. A mini overview of isolation, characterization and application of amniotic fluid stem cells. Int J Stem Cells. 2015;8(2):115-120. doi:10.15283/ijsc.2015.8.2.115

31. Li L, Wang D, Zhou J, Cheng Y, Liang T, Zhang G. Characteristics of human amniotic fluid mesenchymal stem cells and their tropism to human ovarian cancer. PLoS One. 2015;10(4):e0123350. doi:10.1371/journal.pone.0123350

32. Gholizadeh-Ghaleh Aziz S, Fardyazar Z, Pashaei-Asl F, Rahmati-Yamchi M, Khodadadi K, Pashaiasl M. Human amniotic fluid stem cells (hAFSCs) expressing p21 and cyclin D1 genes retain excellent viability after freezing with (dimethyl sulfoxide) DMSO. Bosn J Basic Med Sci. 2019;19(1):43-51. doi:10.17305/bjbms.2018.2912

33. Gholizadeh-Ghaleh Aziz S, Pashaei-Asl F, Fardyazar Z, Pashaiasl M. Isolation, characterization, cryopreservation of human amniotic stem cells and differentiation to osteogenic and adipogenic cells. PLoS One. 2016;11(7):e0158281. doi:10.1371/journal.pone.0158281

34. Lu YR, Yuan Y, Wang XJ, et al. The growth inhibitory effect of mesenchymal stem cells on tumor cells in vitro and in vivo. Cancer Biol Ther. 2008;7(2):245-251. doi:10.4161/ cbt.7.2.5296

35. Magatti M, De Munari S, Vertua E, Parolini O. Amniotic membrane-derived cells inhibit proliferation of cancer cell lines by inducing cell cycle arrest. J Cell Mol Med. 2012;16(9):2208-2218. doi:10.1111/j.15824934.2012.01531.x

36. Gholizadeh-Ghaleh Aziz S, Fardyazar Z, Pashaiasl M. The human amniotic fluid mesenchymal stem cells therapy on, SKOV3, ovarian cancer cell line. Mol Genet Genomic Med. 2019;7(7):e00726. doi:10.1002/mgg3.726

37. Alanazi IO, Al Shehri ZS, EbrahimieE, Giahi H, MohammadiDehcheshmeh M. Non-coding and coding genomic variants distinguish prostate cancer, castration-resistant prostate cancer, familial prostate cancer, and metastatic castrationresistant prostate cancer from each other. Mol Carcinog. 2019;58(6):862-874. doi:10.1002/mc.22975

38. Pashaei-Asl F, Pashaei-Asl R, Khodadadi K, Akbarzadeh A, Ebrahimie E, Pashaiasl M. Enhancement of anticancer activity by silibinin and paclitaxel combination on the ovarian cancer. Artif Cells Nanomed Biotechnol. 2018;46(7):14831487. doi:10.1080/21691401.2017.1374281

39. Pashaiasl M, Khodadadi K, Kayvanjoo AH, Pashaei-Asl R,
Ebrahimie E, Ebrahimi M. Unravelling evolution of Nanog, the key transcription factor involved in self-renewal of undifferentiated embryonic stem cells, by pattern recognition in nucleotide and tandem repeats characteristics. Gene. 2016;578(2):194-204. doi:10.1016/j.gene.2015.12.023

40. Pashaiasl M, Ebrahimi M, Ebrahimie E. Identification of the key regulating genes of diminished ovarian reserve (DOR) by network and gene ontology analysis. Mol Biol Rep. 2016;43(9):923-937. doi:10.1007/s11033-016-4025-8

41. You Q, Cai L, Zheng J, Tong X, Zhang D, Zhang Y. Isolation of human mesenchymal stem cells from third-trimester amniotic fluid. Int J Gynaecol Obstet. 2008;103(2):149-152. doi:10.1016/j.ijgo.2008.06.012

42. Gholizadeh-Ghaleh Aziz S, Fathi E, Rahmati-Yamchi M, Akbarzadeh A, Fardyazar Z, Pashaiasl M. An update clinical application of amniotic fluid-derived stem cells (AFSCs) in cancer cell therapy and tissue engineering. Artif Cells Nanomed Biotechnol. 2017;45(4):765-774. doi:10.1080/216 91401.2016.1216857

43. Ramdasi S, Sarang S, Viswanathan C. Potential of mesenchymal stem cell based application in cancer. Int J Hematol Oncol Stem Cell Res. 2015;9(2):95-103.

44. Liu J, Han G, Liu H, Qin C. Suppression of cholangiocarcinoma cell growth by human umbilical cord mesenchymal stem cells: a possible role of Wnt and Akt signaling. PLoS One. 2013;8(4):e62844. doi:10.1371/journal.pone.0062844

45. Ohta N, Ishiguro S, Kawabata A, et al. Human umbilical cord matrix mesenchymal stem cells suppress the growth of breast cancer by expression of tumor suppressor genes. PLoS One. 2015;10(5):e0123756. doi:10.1371/journal.pone.0123756

46. Yuan Y, Zhou C, Chen X, Tao C, Cheng H, Lu X. Suppression of tumor cell proliferation and migration by human umbilical cord mesenchymal stem cells: A possible role for apoptosis and Wnt signaling. Oncol Lett. 2018;15(6):85368544. doi:10.3892/ol.2018.8368

47. Sierra-Torres CH, Tyring SK, Au WW. Risk contribution of sexual behavior and cigarette smoking to cervical neoplasia. Int J Gynecol Cancer. 2003;13(5):617-625.

48. Juneja A, Sehgal A, Mitra AB, Pandey A. A survey on risk factors associated with cervical cancer. Indian J Cancer. 2003;40(1):15-22.

49. Tomaic V. Functional roles of E6 and E7 oncoproteins in HPV-induced malignancies at diverse anatomical sites. Cancers (Basel). 2016;8(10):95. doi:10.3390/cancers8100095

50. Zhai Y, Kuick R, Nan B, et al. Gene expression analysis of preinvasive and invasive cervical squamous cell carcinomas identifies HOXC10 as a key mediator of invasion. Cancer Res. 2007;67(21):10163-10172. doi:10.1158/0008-5472.can07-2056

51. el-Deiry WS, Tokino T, Velculescu VE, et al. WAF1, a potential mediator of p53 tumor suppression. Cell. 1993;75(4):817825. doi:10.1016/0092-8674(93)90500-p

52. el-Deiry WS, Harper JW, O'Connor PM, et al. WAF1/CIP1 is induced in p53-mediated G1 arrest and apoptosis. Cancer Res. 1994;54(5):1169-1174.

53. Raisova M, Hossini AM, Eberle J, et al. The Bax/Bcl-2 ratio determines the susceptibility of human melanoma cells to CD95/Fas-mediated apoptosis. J Invest Dermatol. 2001;117(2):333-340. doi:10.1046/j.0022-202x.2001.01409.x

(c) 2020 The Author(s); This is an open-access article distributed under the terms of the Creative Commons Attribution License (http:// creativecommons.org/licenses/by/4.0), which permits unrestricted use, distribution, and reproduction in any medium, provided the original work is properly cited. 